\title{
Caesarean Section Without Using Bladder Catheterization Is Safe in Uncomplicated Patients
}

\author{
Amat-Al Karem Ali Hamad Al Huri ${ }^{1}$, Athmar Hussein Ali ${ }^{1}$, Abdelrahman Hasan Al Harazi ${ }^{2}$ * \\ ${ }^{1}$ Department of Obstetrics and Gynecology, Faculty of Medicine, Sana'a University, Sana'a, Yemen \\ ${ }^{2}$ Department of Obstetrics and Gynecology, Faculty of Medicine, Thamar University, Sana'a, Yemen
}

Email address:

osama.m.alshami@gmail.com (Amat-Al K. A. H. Al H.), Athmarali265@gmail.com (Athmar H. A.), alharazi77184@hotmail.com (Abdelrahman H. Al H.)

${ }^{*}$ Corresponding author

\section{To cite this article:}

Amat-Al Karem Ali Hamad Al Huri, Athmar Hussein Ali, Abdelrahman Hasan Al Harazi. Caesarean Section Without Using Bladder Catheterization Is Safe in Uncomplicated Patients. Journal of Gynecology and Obstetrics. Vol. 5, No. 5, 2017, pp. 56-59.

doi: 10.11648/j.jgo.20170505.11

Received: July 17, 2017; Accepted: July 24, 2017; Published: August 14, 2017

\begin{abstract}
A case - control study was carried out to assess whether carrying out caesarean section without urethral catheterization is safe in terms of intraoperative safety, prevalence of UTI, and the rate of the first voiding discomfort. It was conducted At Al Thawra General Hospital over a period of eight months (From Feb $1^{\text {st }}$ to Sep $30^{\text {th }} 2015$ ). One hundred women who admitted to delivery unit and delivered by cesarean section were enrolled in the study. They were divided randomly into study group (non catheterized, $\mathrm{n}=50$ ) and control group (catheterized, $\mathrm{n}=50$ ). There were no significant differences between the study and control group regarding maternal age, parity and the indications for cesarean section. Of the study group, only one women developed bladder distension at the end of surgery. First voiding discomfort was detected among $86 \%$ of the control group vs. $50 \%$ of the study cases $(P<0.05)$. The mean time till ambulation was $8.2 \pm 2.1 \mathrm{~h}$ for the study group compared to $14.1 \pm 2.9 \mathrm{~h}$ for the control group $(P<0.001)$. Urinary tract infection was significantly higher among the control group (28\%) vs $(8 \%)$ of the study cases $(P=0.009)$. This study showed that nonuse of urinary catheter during cesarean section is associated with significantly low rate of UTI, less first voiding discomfort and early ambulation. Therefore, urinary catheter can be avoided safely in hemodynamecally stable patients.
\end{abstract}

Keywords: Cesarean Section, Urethral Catheterization, Urinary Tract Infection

\section{Introduction}

For more than 3500 years, urinary catheters have been used to drain the bladder when it fails to empty. The modern balloon - based, self - retaining catheter designed by Frederic Foley has marketed in 1933 [1]. An indwelling urinary catheter is a routine part of most surgeries including cesarean section (CS) performed today, inserted prior to surgery and remains $12-24 \mathrm{~h}$ post operation [2]. The reasons cited are: better bladder exposure during surgery, decreased risk of intraoperative injury to the urinary system, urine output assessment, and prevention of postoperative urinary retention [3]. However, catheterization of the urinary tract during $\mathrm{CS}$ has been implicated as a main cause of urinary tract infection (UTI) [4]. In addition, the practice of catheterization imposes the cost of urinary catheter, urine bags and antimicrobial therapy for UTI besides patients discomfort and delayed ambulation [4]. Genitourinary infection is one of the most complications of CS, accounting for greater than $80 \%$ of nosocomial UTI and greater postoperative pain [5]. Bacteriuria develops in about $10-15 \%$ of the hospitalized patients with indwelling urinary catheter and the risk of infection is approximately 3 $-5 \%$ per day of catheterization [6].

Several studies have been shown that CS performed without using urethral catheter as save as the traditional approach [7]. Instead, some additional benefits have been reported such as low rate of UTI, less voiding discomfort, early ambulation and shorter hospital stay [8]. The aim of this study was to assess whether carrying out CS without 
urethral catheterization is safe in terms of intraoperative safety, prevalence of UTI, and the rate of the first voiding discomfort.

\section{Patient and Methods}

This is a cross - sectional study carried out at Al Thawra General Hospital, over eight months (From Feb $1^{\text {st }}$ to Sep $\left.30^{\text {th }} 2015\right)$. We included in this study 100 pregnant women with gestational age $\geq 38$ weeks who admitted to labor ward for elective, repeat or emergency cesarean section. We excluded from this study all women who had hypertensive disorders of pregnancy, antepartum hemorrhage, prior two or more scars, cardiovascular, renal or liver disease. Written consent was obtained from each subject and the study protocol was approved by the ethics committee of the hospital. The participants were divided randomly into two groups, the study group (uncatheterized, $\mathrm{UG}, \mathrm{n}=50$ ) and control group (catheterized, $\mathrm{CG}, \mathrm{n}=50$ ). We used the simple randomization procedure as for each uncatheterized women, the next women matched with age, parity, and indication for CS was selected as a control. After admission, detailed history, physical examination and investigation were performed for all women. The investigations included complete blood count, white blood cells with differential and urinalysis. The data were filled in a questionnaire. After standard preoperative preparation for both groups, Foley's catheter was inserted for the control group. All subjects of the study group were asked to void immediately before operation. Cesarean sections were performed under spinal anesthesia and lower uterine segment transverse incision was made for all patients. After delivery of the baby, all patients in both groups received a single dose of intravenous cefazolin $2 \mathrm{~g}$ after sensitivity test, and 20 units of oxytocin in $500 \mathrm{ml}$ Ringer lactate solution. The rest of the procedure was completed as standard. Cesarean sections were performed by the senior in - charge who did not know the parameters measured. Patients who developed intraoperative complications such as bleeding more than usual or uterine atony, Foley's catheter was inserted and the patient was excluded from the study. Likewise, patients who developed postpartum hemorrhage, similar steps were taken. After each $\mathrm{CS}$, the surgeon received questionnaire to explain whether he / she encountered intraoperative difficulties. Postoperative care was followed as usual including closed monitoring of vitals, analgesic, nothing per month for the first 8 hours. Patients of study group were asked to report urge to void for the first time and helped by bedpan when necessary. The first voiding time was defined as the interval between the onset of surgery and first spontaneous voiding. Discomfort in the first voiding was defined as burning, urging or difficulty to void. The level of pain was categorized subjectively as no pain, mild pain and moderate to severe pain. The time of first ambulation was defined as the interval between the onset of surgery and the time of patient first ambulated. Foley's catheter was removed from CG in the morning after surgery. Urine for both groups was sampled $48 \mathrm{~h}$ post operation and subjected to culture and sensitivity. Patients who had positive results, the appropriate antibiotic was given. The outcome measures were the frequency of UTI, frequency of the first voiding discomfort, safety of cesarean section without catheterization, and hospital stay.

Statistical Analysis

All statistical analysis were performed using SPSS 21 (SPSS Inc., Chicago, IL, USA) statistical programs. Independent $t$ test was applied for the test of significance of the numerical data. Ordinal data was assessed with the Chisquare and Fischer's exact test. A $P$ value $<0.05$ was considered statistically significance.

\section{Results}

There were 50 women in each group. Women in both groups had no differences in age, parity, cesarean section indications and received the same pre and post operation care (Table 1$)$. Only one woman (2\%) of UG developed bladder distension at the end of operation. The first voiding time observed among UG occurred between $4-8 \mathrm{~h}$ in (64\%), and in $(36 \%)$ after $8 \mathrm{~h}$. Only ( $8 \%$ ) needed bedpan. Discomfort at the first voiding was noted in $(86 \%)$ of $\mathrm{CG}$ compared to $(50 \%)$ of the UG. The difference was statistically significant $(P<0.05)$. Among CG, the level of discomfort was mild in $64 \%$, while $22 \%$ experienced moderate to severe pain, compared to $42 \%$ and $8 \%$ for UG respectively. The differences between the two groups were statistically significant $(P<0.05)$. The mean time till ambulation for UG was $8.2 \pm 2.1 \mathrm{~h}$ versus $14.1 \pm 2.9 \mathrm{~h}$ for $\mathrm{CG}$. The difference was statistically significant $(P<0.0001)$. There was no significant difference between the two groups regarding mean operating time $(42.6 \pm 5.3 \mathrm{~min}$ and $43.1 \pm 5.6 \mathrm{~min})$ for $\mathrm{UC}$ and $\mathrm{CG}$ respectively.

Catheterization was needed for 2 cases, the first case catheter was inserted at the end of CS due to bladder distension whereas the another case, catheter was inserted during the first post operation day because of urine retention.

The incidence of UTI was $28 \%$ for the CG. The difference was statistically significant $(P=0.009)$. There was no significant difference between the two groups in febrile morbidity $(P=0.30)$. Significantly shorter among UG $(\mathrm{P}=$ 0.04). Table 2 summarizes the outcome results.

Table 1. Patients' obstetric characteristics.

\begin{tabular}{llll}
\hline Variable & UC $(\mathbf{n}=\mathbf{5 0})$ & CG (n= 50) & P value \\
\hline Age $(\mathrm{y})$ & $26.3 \pm 4.5$ & $25.9 \pm 5.1$ & 0.69 \\
Parity & & & \\
1 & $14(28)$ & $12(24)$ & 0.65 \\
$2-4$ & $30(60)$ & $33(66)$ & 0.53 \\
$\geq 5$ & $6(12)$ & $5(10)$ & 0.75 \\
\hline
\end{tabular}




\begin{tabular}{llll}
\hline Variable & $\mathbf{U C}(\mathbf{n}=\mathbf{5 0})$ & $\mathbf{C G}(\mathbf{n}=\mathbf{5 0})$ & $\boldsymbol{P}$ value \\
\hline Indications for cesarean & & & 0.56 \\
Malpresentation & $8(16)$ & $6(12)$ & 0.78 \\
Prior one CS & $7(14)$ & $8(16)$ & 0.76 \\
Multiple pregnancy & $6(12)$ & $7(14)$ & 0.79 \\
Failure to progress & $10(20)$ & $9(18)$ & \\
Elective & $11(22)$ & $10(20) 0.80$ & \\
Others & $8(16)$ & $10(20) 0.60$ & \\
\hline
\end{tabular}

Data expressed as mean $\pm \mathrm{SD}$ or $\mathrm{n}(\%)$.

CS: Caesarean section. UC: non-catheterized group; CG: Catheterized group.

Table 2. Outcome measures of the study population.

\begin{tabular}{|c|c|c|c|}
\hline Variable & $\mathrm{UC}(\mathrm{n}=50)$ & CG $(n=50)$ & $P$ value \\
\hline Bladder distension & $1(2)$ & - & \\
\hline Mean operating time, min. & $42.6 \pm 5.3$ & $43.1 \pm 5.6$ & 0.64 \\
\hline \multicolumn{4}{|l|}{ First voiding time, $\mathrm{h}$} \\
\hline$>8$ & $18(36)$ & - & \\
\hline \multicolumn{4}{|l|}{ First voiding discomfort } \\
\hline None & $25(50)$ & $7(14)$ & 0.00 \\
\hline Moderate - severe & $4(8)$ & $11(22)$ & 0.05 \\
\hline Time till ambulation, $\mathrm{h}$ & $8.2 \pm 2.1$ & $14.1 \pm 2.9$ & $<0.001$ \\
\hline Catheterization at or post CS & $2(4)$ & - & \\
\hline Urine retention & $1(2)$ & - & \\
\hline Febrile morbidity & $1(2)$ & $3(6)$ & 0.30 \\
\hline Positive urine culture, $48 \mathrm{~h}$ & $4(8)$ & $14(28)$ & 0.009 \\
\hline Hospital stay, day & $2.9 \pm 1.4$ & $3.7 \pm 1.1$ & 0.04 \\
\hline
\end{tabular}

Data expressed as mean $\pm \mathrm{SD}$ or $\mathrm{n}(\%)$

UC: non-catheterized group; CG: Catheterized group.

\section{Discussion}

This study showed that when cesarean section carried out without using urethral catheterization, there was no increased risk of bladder distension that may interfere with surgical exposure to the lower uterine segment and could possibly result in the urinary tract injury. Only one case of UG developed bladder distension at the end of surgery. This finding is similar to other studies $[9,4]$. The low rate of urinary bladder distension detected in our study may be related to short operation time as well as to voiding immediately before operation. Consequently, the intraoperative difficulty was not seen and the operative field was easily exposed after retraction of the urinary bladder with Doyen's retractor. Even when the bladder distension develops at the start of surgery, we support Nasre et al argument that a slightly filled bladder may be better demarcated and therefore, more easily identified intraoperatively [9].

Our results showed significantly higher incidence of UTI among CG (28\% versus $8 \%, \mathrm{P}<0.005)$. This finding is comparable to Pandey D (2015) who found among 75 women assigned for catheterization that the incidence of UTI detected by urine culture was $29.3 \%$ compared to $4 \%$ of UG, $(\mathrm{P}<0.005)[10]$, but higher than that reported by Nasre AM et al [9] who found the incidence of UTI among catheterized women $(\mathrm{n}=210)$ by simple urine analysis as $5.7 \%$ and $2.9 \%$ after $24 \mathrm{~h}$ and 1 week respectively. This could be explained by small sample size of our study.
This study found a higher incidence of the first voiding discomfort experienced among CG. Although the degree of discomfort was mild in the majority of patients, the difference between the two groups was statistically significant $(\mathrm{P}<0.005)$. This results are in agreement with the results observed by Arlyn E [11], Nasre AM (9) and Pandey $\mathrm{D}[10]$.

It is observed in this study that patients with noncatheterized urinary bladder (UG) initiated ambulation significantly earlier than those in CG, similar to other studies $[4,9,10]$. It is likely that early ambulation could be attributed to the absence of urinary catheter, which restricts patients' movements, and fear of accidental expulsion [12].

The role of CS in urinary retention is difficult to delineate due to the effects of anesthesia and operation both causing postpartum bladder changes $[13,14]$. The paralyzing effect of anesthesia especially conduction analgesia and the temporarily disturbed bladder neural function are undoubtedly contributing factor [15]. In the UK, the National Institute for Health and Clinical Excellence recommends that women having caesarean section with regional anesthesia should have an indwelling urinary catheter to prevent over distension of the bladder, because the anesthetic block interferes with normal bladder function [16]. In our study, we found nonuse of urinary catheterization during cesarean section was not associated with an increase in urinary retention.

Only one patient developed urinary retention $(2 \%)$, similar to other studies $[9,4]$, but much lower than the observed 
range of $3.3-39.2 \%$ [17]. It is reported that the low rate of postoperative urine retention among UG could be the result of adequate analgesia, early ambulation and re catheterization in $\mathrm{UG}$ when needed $[18,7,9,4]$.

Although our results found a significant benefits of non use of urinary bladder catheter, the decision whether to catheterize should be based on selection criteria including the indication for CS, type of anesthesia and the patient's medical profile as hemodynamically unstable patients should have urinary catheter placement.

The limitations of the study are: small sample size that could limit the generalizability of the results. Another limitation is that we used spinal anesthesia for all CS operations, so the other types may have different effects on intra and postoperative bladder function. In addition, we did not study the costs of both urinary catheter and antimicrobial drugs because they offered free to all patients.

\section{Conclusion}

This study demonstrated that nonuse of urinary catheter during cesarean section is associated with significantly low rate of UTI, less first voiding discomfort, early ambulatio, and therefore early discharge. There were no significant difference in the mean operation time between the two groups and urinary retention was not increased. These results suggest that urinary catheter can be avoided safely in hemodynamically stable patients. However, larger studies are needed to support our findings.

\section{References}

[1] M 1 Feneley RC, Hopley IB, Wells PN. Urinary catheters: history, current status, adverse events and research agenda. J Med Eng Technol 2015; 39: 459-70.

[2] Lang JF, Bowen JC, Strong P. Use of indwelling urinary catheter at cesarean delivery. Obstetrics \& Gynecology. 2001 Apr 1; 97 (4): S 66.

[3] Onyegbule O A, Udigwe G O, Ezebialu I, Nduka A C, Okolie $\mathrm{V}$ E and Okor O L. Catheter-Associated Urinary Tract Infection Following Caesarean Section in Nnewi, Nigeria: A Prospective Comparative Study British Microbiology Research Journal 4(9): 1025-1034, 2014.

[4] Ghorieshi J. Indwelling urinary catheters in cesarean delivery. Int J Gynecol Obstet. 2003; 83 (3): 267-70.
[5] Ercole FF, Macieira TG, Wenceslau LC, Martins AR, Campos $\mathrm{CC}$, Chianca TC. Integrative review: evidences on the practice of intermittent/indwelling urinary catheterization. Revista latino-americana de enfermagem. 2013 Feb; 21 (1): 459-68.

[6] Kasper DL, Braunwald E, Fauci AS, Hauser SL, Longo DL. Harrison's principles of internal medicine. 16th ed. McGrawHill Publishers; 2005: 1717-18.

[7] Senanayake H. Elective cesarean section without urethral catheterization. J Obstet. Gynaecol 2005; 31: 32-7.

[8] Acharya S, Uprety DK, Pokharel HP, Amatya R, Rai R. Cesarean section without urethral catheterization: a randomized control trial. Kathmandu University Medical Journal. 2013 Jan 3; 10 (2): 18-22.

[9] Nasr AM, Elbigawy AF, Abdelamid AE, Al-Khulaldis, AlInany HG, Sayed EH. Evaluation of the use Vs nonuse of urinary catheterization during caesarean delivery: A prospective, multicenter, randomized controlled trial. $J$ Perinatol. 2009; 29: 416-21.

[10] Pandey D, Mehta S, GroverA, Goel N. Indwelling Catheterization in Caesarean Section: Time To Retire It! Journal of Clinical and Diagnostic Research. 2015, 9 (9): 1-4.

[11] Arlyn E, Antonia E. Habana. Early removal of urinary catheter in caesarean delivery in a tertiary training hospital. Philippine journal of Obstetrics \& Gynecology. 2007; 31 (2): 69-74.

[12] Beeson PB. The case against the catheter. Am J Med. 2000; 24 : $1-6$.

[13] Liang CC, Wu MP, Chang YL, Chueh HY, Chao AS, Chang SD. Voiding dysfunction in women following cesarean delivery. Taiwanese Journal of Obstetrics and Gynecology. 2015 Dec 31; 54 (6): 678-81.

[14] Darrah DM, Griebling TL, Silverstein JH. Postoperative urinary retention. Anesthesiology clinics. 2009 Sep 30; 27 (3): 465-84.

[15] Rochelle L In: Cunningham FG, MacDonald PC, Gant NF (eds). Williams Obstetrics. 22nd edn. Vol 23 McGraw-Hill: New York, 2005, pp 523.

[16] National Institute for Clinical Excellence. Caesarean section [guidan ce.nice.org.uk/CG 13]. Last accessed 10 September 2016.

[17] Humburg J. Postpartum urinary retention - without clinical impact? Ther Umsch 2008; 65: 681-5.

[18] Li L, Wen J, Wang $\mathrm{L}$, et al. Is routine indwelling catheterisation of the bladder for caesarean section necessary? A systematic review. BJOG 2011; 118: 400. 